\title{
Glucosinolates in Chinese Brassica campestris Vegetables: Chinese Cabbage, Purple Cai-tai, Choysum, Pakchoi, and Turnip
}

\author{
Xinjuan Chen \\ Department of Horticulture, Zhejiang University, Kaixuan Road 268, \\ Hangzhou 310029, China; and the Institute of Vegetable, Zhejiang \\ Academy of Agriculture Science, Hangzhou 310021, China \\ Zhujun Zhu' \\ Department of Horticulture, Zhejiang University, Kaixuan Road 268, Hangzhou \\ 310029, China; and the Department of Horticulture, School of Agriculture \\ and Food Science, Zhejiang Forestry University, Lin'an 311300, China
}

\section{Joska Gerendás and Nadine Zimmermann \\ Institute for Plant Nutrition and Soil Science, University of Kiel, Olshausenstr. 40, Kiel 24098, Germany}

Additional index words. Chinese vegetable, glucosinolate, Brassicaceae, composition

\begin{abstract}
Brassica campestris vegetables play an important role in the Chinese diet. The objective of this study was to evaluate the composition and content of glucosinolates (GSs) in five species of Chinese Brassica campestris vegetables by high-performance liquid chromatography. The compositions and contents of GSs varied significantly among and within species and cultivars. The contents of total GSs were 100 to $130 \mathrm{mg} / 100 \mathrm{~g}$ fresh weight (FW) in turnip (B. rapifera), 50 to $70 \mathrm{mg} / 100 \mathrm{~g} \mathrm{FW}$ in purple cai-tai $(B$. chinensis var. purpurea), and 14 to $35 \mathrm{mg} / 100 \mathrm{~g} \mathrm{FW}$ in Chinese cabbage (B. pekinensis), choysum (B. chinensis var. utilis), and pakchoi (B. chinensis var. communis). In Chinese cabbage, the predominant individual GSs were glucobrassicin for both cultivars, neoglucobrassicin only for 'zaoshuwuhao', and gluconapin only for 'zaoshuwuhao'. The predominant individual GSs were glucobrassicanapin and gluconapin in purple cai-tai and choysum and gluconapin in pakchoi and turnip. The relative content of total aliphatic GSs was $80 \%$ to $90 \%$ in purple cai-tai and choysum, $60 \%$ to $65 \%$ in pakchoi and turnip, and $17 \%$ to $50 \%$ in Chinese cabbage. The relative content of total indolic GSs was $37 \%$ to $\mathbf{7 5 \%}$ in Chinese cabbage, $25 \%$ to $27 \%$ in pakchoi, and $5 \%$ to $17 \%$ in purple caitai, choysum, and turnip. The relative content of aromatic GSs was $28 \%$ to $36 \%$ in turnip, $8 \%$ to $14 \%$ in Chinese cabbage and pakchoi, and $2 \%$ to $4 \%$ in choysum and purple cai-tai. These results suggest that there are significant genotypic variations in composition and content of glucosinolates in Chinese Brassica campestris vegetables.
\end{abstract}

Epidemiological data show that a diet rich in cruciferous vegetables can reduce the risk from a number of cancers. Several protection mechanisms for the cancer prevention from cruciferous vegetables have been demonstrated for the breakdown products of some glucosinolates (GSs) (Mithen et al., 2000). Some research about glucosinolates has been done in Brassica crops (Carlson et al., 1987; Krumbein et al., 2005; Mullin and Sahasrabudhe, 1977; Rangkadilok et al., 2002; Rosa et al., 1996; Sang et al., 1984). Brassica campestris vegetables play an important role in the Chinese diet, and so their naturally occurring GSs in edible parts should be moni-

Received for publication 21 Oct. 2006. Accepted for publication $10 \mathrm{Feb} .2007$.

This research was financially supported by SinoGerman Center for Science Promotion [Grant GZ 051/10 (154)] and the German Research Foundation. ${ }^{1}$ To whom reprint requests should be addressed; e-mail zhjzhu@zju.edu.cn tored. To our knowledge, there is limited information about the comparison of GSs among Chinese Brassica campestris vegetables (He et al., 2000, 2002; Hill et al., 1987; Lewis and Fenwick, 1988). Therefore, the objective of this study was to compare and evaluate the composition and content of GSs in edible parts in five species of Chinese Brassica vegetables, including Chinese cabbage (Brassica campestris L. ssp. pekinensis), choysum (Brassica campestris L. ssp. chinensis var. utilis), purple cai-tai (Brassica campestris L. ssp. chinensis var. purpurea), pakchoi (Brassica campestris L. ssp. chinensis var. communis), and turnip (Brassica campestris L. ssp. rapifera).

\section{Materials and Methods}

Plant materials. Five species of Brassica campestris vegetables were used for this experiment. The materials used in this exper- iment are shown in Table 1. Seeds were sown on 15 Sept. 2004 and transplanted at the farm of Zhejiang University on 15 Oct. 2004 at a randomized complete block design with three replicates of 20 plants each. For analysis, the edible parts of five plants were harvested at commercial maturity stage from each of the three replicates (see Table 1). Edible parts (50 to $200 \mathrm{~g}$ of each replicate) were immediately deep frozen with liquid nitrogen, then freeze-dried, and finely ground. The production period and the climatic conditions under a field conditions are shown in Table 1 .

Methods. Samples were prepared according to the method of Krumbein et al. (2005) with slight modification. Duplicates of the freeze-dried powder $(0.25 \mathrm{~g})$ in 10 -mL glass tubes were preheated for $5 \mathrm{~min}$ in $75^{\circ} \mathrm{C}$ water bath. Four milliliters of $70 \%$ boiling methanol $\left(75^{\circ} \mathrm{C}\right)$ was added and extracted at $75^{\circ} \mathrm{C}$ in a water bath for $10 \mathrm{~min}$. There was $100 \mu \mathrm{L}$ of $5 \mathrm{mmol} \cdot \mathrm{L}^{-1}$ sinigrin (Sigma-Aldrich Co., St. Louis) as an internal standard in one of the duplicates before extraction. Then $1 \mathrm{~mL}$ of $0.4 \mathrm{~mol} \cdot \mathrm{L}^{-1}$ barium acetate was rapidly added and vortexed for several seconds. After centrifugation at $4000 \mathrm{rpm}$ for $10 \mathrm{~min}$ at room temperature, the supernatants were collected and the pellets were reextracted twice with $3 \mathrm{~mL}$ of $70 \%$ boiling methanol $\left(75^{\circ} \mathrm{C}\right)$. Three supernatants were combined and made into a final volume of $10 \mathrm{~mL}$ with $70 \%$ methanol. Five-milliliter extracts were loaded onto a 1-mL mini-column (JT Baker, Phillipsburg, PA) prepared by introducing $500 \mu \mathrm{L}$ of activated DEAE Sephadex A25 (Amersham Biosciences, Uppsala, Sweden) in a vacuum processor (JT Baker 12) and allowed to desulphate overnight with aryl sulfatase (Sigma-Aldrich Co.). The resultant desulpho (ds)-GS were eluted with $2.5 \mathrm{~mL}$ of ultra pure water produced by Milli-Q system (Millipore Co., Milford, CT) and stored at $-20{ }^{\circ} \mathrm{C}$ before separation by high-performance liquid chromatography (HPLC).

The elution $(20 \mu \mathrm{L})$ was analyzed in a Shimadzu HPLC system (LC-10AT pump, CTO-10A column oven, SCL-10A VP system controller; Shimadzu, Kyoto, Japan) consisting of an ultraviolet-VIS detector (SPD-10A) set at $229 \mathrm{~nm}$ and a prontosil ODS2 column $(250 \times 4 \mu \mathrm{m}, 5 \mu \mathrm{m}$; Bischoff, Leonberg, Germany). The mobile phase was ultra pure water (A) and acetonitrile (Tedia, Fairfield, OH) (B) in a linear gradient from $0 \%$ to $20 \%$ B for $32 \mathrm{~min}$, then constant $20 \% \mathrm{~B}$ for $6 \mathrm{~min}$, and $100 \% \mathrm{~B}$ and $0 \% \mathrm{~B}$ before the injection of the next sample. The flow rate was $1.3 \mathrm{~mL} \cdot \mathrm{min}^{-1}$. Each individual $\mathrm{ds}-\mathrm{GS}$ was identified in the HPLC system coupled with an electrospray ionization ion trap mass detector system (Agilent 1100 series, Agilent Technologies, Palo Alto, CA). The HPLC conditions were the same as described previously, except the flow rate was $1.0 \mathrm{~mL} \cdot \mathrm{min}^{-1}$. The nebulizer pressure is $60 \mathrm{psi}$ and the flow rate is nitrogen $13 \mathrm{~mL} \cdot \mathrm{min}$ at a drying temperature of $350{ }^{\circ} \mathrm{C}$. The scan of the masses ranged from $100 \mathrm{~m} / \mathrm{z}$ to $600 \mathrm{~m} / \mathrm{z}$ and helium was used as collision gas for the fragmentation procedure of the isolated compounds in 
Table 1. Species, cultivars, and edible parts of five Brassica campestris vegetables and the production period and climatic conditions.

\begin{tabular}{|c|c|c|c|c|c|}
\hline Common name & Latin name & Cultivars & Edible part & Production period & $\begin{array}{l}\text { Avg day/night } \\
\text { temp }\left({ }^{\circ} \mathrm{C}\right)\end{array}$ \\
\hline Chinese cabbage & B. campestris ssp. pekinensis & Huangyacai Zaoshuwuhao & Leaves & 15 Sept. to 10 Dec. & $21.6 / 12.7$ \\
\hline Purple cai-tai & $\begin{array}{l}\text { B. campestris ssp. chinensis } \\
\text { var. purpurea }\end{array}$ & Wushihong and Xiangyanhong & $\begin{array}{l}\text { Bolting stems } \\
\text { and inflorescences }\end{array}$ & 15 Sept. to 20 Nov. & $23.3 / 14.6$ \\
\hline Choysum & $\begin{array}{l}\text { B. campestris ssp. chinensis } \\
\text { var. utilis }\end{array}$ & Youqing and Sijiu & $\begin{array}{l}\text { Bolting stems } \\
\text { and inflorescences }\end{array}$ & 15 Sept. to 20 Nov. & $23.3 / 14.6$ \\
\hline Pakchoi & $\begin{array}{l}\text { B. campestris ssp. chinensis } \\
\text { var. communis }\end{array}$ & Huqing and Wuyoudong & Leaves & 15 Sept. to 20 Nov. & 23.3/14.6 \\
\hline Turnip & B. campestris ssp. rapifera & Wenzhoubai and Wenzhouhong & Roots & 15 Nov. to 10 Dec. & $21.6 / 12.7$ \\
\hline
\end{tabular}

the ion trap. The ionization condition of capillary voltage was $4000 \mathrm{~V}$. The mass detection was recorded in the positive modus. Each individual GS was identified according to their $(\mathrm{M}+\mathrm{H})^{+},(\mathrm{M}+\mathrm{Na})^{+},(\mathrm{M}+\mathrm{K})^{+}$, and $(\mathrm{M}-$ glucosyl $+\mathrm{H})^{+}$. The amounts of each GS were calculated based on the published ultraviolet response factors of other ds-GS relative to ds-sinigrin (European Community, 1990).

Data analysis. Differences between means were analyzed by Fisher's protected least significant difference procedure.

\section{Results and Discussion}

Total glucosinolates. There were significant differences in total GSs among and within these Brassica campestris vegetables (Fig. 1). The contents of total GSs in turnip were the highest ranging from $102.43 \mathrm{mg} / 100$ $\mathrm{g}$ fresh weight (FW) to $124.57 \mathrm{mg} / 100 \mathrm{~g} \mathrm{FW}$, followed by purple cai-tai $(50$ to $70 \mathrm{mg} / 100 \mathrm{~g}$ FW), and the lowest in Chinese cabbage, choysum, and pakchoi (14 to $35 \mathrm{mg} / 100 \mathrm{~g}$ FW). This result was consistent with that of Carlson et al. (1987) who reported the considerable differences in total GS levels among and within seven Brassica species. There was consistent reports that total GS content was $198 \mathrm{mg} / \mathrm{kg} \mathrm{FW}$ in Chinese cabbage and 534 $\mathrm{mg} / \mathrm{kg} \mathrm{FW}$ in pakchoi (Lewis and Fenwick, 1988). He et al. (2002) reported the content of total GSs was $71.49 \mu \mathrm{mol} / 100 \mathrm{~g} \mathrm{FW}(29.17$ $\mathrm{mg} / 100 \mathrm{~g} \mathrm{FW}$ ) in pakchoi and $295.65 \mu \mathrm{mol} /$ 100 g FW (120.63 mg/100 g FW) in choysum. Although the same edible parts were analyzed, the differences in total GSs of Chinese cabbage, pakchoi, and choysum in different experiments may be caused by the variety, plant age, and the growing conditions.

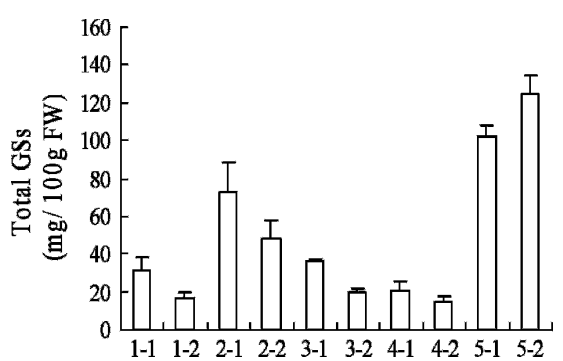

Fig. 1. Total glucosinolates in Chinese Brassica campestris vegetables 1-1 Chinese cabbage 'Huangyacai'; 1-2 Chinese cabbage 'Zaoshuwuhao'; 2-1 Purple cai-tai 'Wushihong'; 2-2 Purple cai-tai 'Xiangyanhong'; 3-1 Choysum 'Youqing'; 3-2 Choysum 'Sijiu'; 4-1 Pakchoi 'Huqing'; 4-2 Pakchoi 'Wuyoudong'; 5-1 Turnip 'Wenzhoubai'; and 5-2 Turnip 'Wenzhouhong'.
Aliphatic, indolic, and aromatic glucosinolates. According to the chemical structure, different GSs can fall into three principal groups comprising the aliphatic group, indolic group, and aromatic group. Except for the difference in total GS levels, there was also considerable variation in the composition and content of aliphatic, indolic, and aromatic GSs (Table 2). Turnip and purple caitai had the highest content of total aliphatic GSs (42 to $73 \mathrm{mg} / 100 \mathrm{~g} \mathrm{FW}$ ); Chinese cabbage and purple caitai 'Wushihong' had the highest content of total indolic GSs (11 to $13 \mathrm{mg} / 100 \mathrm{~g} \mathrm{FW}$ ); and turnip had the highest content of aromatic GSs (29 to $46 \mathrm{mg} / 100 \mathrm{~g} \mathrm{FW}$ ). The relative content of three principal GS groups also can illuminate the differences in GS levels among and within these Brassica campestris vegetables (Table 3). For example, the relative content of total aliphatic GSs in purple caitai and choysum were $80 \%$ to $90 \%, 60 \%$ to $65 \%$ in pakchoi and turnip, and $17 \%$ to $50 \%$ in Chinese cabbage. Furthermore, the relative content of aromatic GSs in turnip and indolic GSs in Chinese cabbage were markedly higher than in other Brassica campestris. This result was consistent with those of Lewis and Fenwick (1988) and He et al. (2002).

Individual glucosinolates. The content of total GSs could not predict the levels of individual GSs. There were greater differences in the levels of individual GSs than total GSs and three principal GS groups. Twelve kinds of individual GSs, including seven aliphatic, four indolic, and one aromatic GSs, were detected. The variation of composition of GSs mainly occurred in aliphatic GSs. For example, Chinese cabbage 'Huangyacai' contained seven kinds of individual GSs and pakchoi 'Wuyoudong' only contained four individual GSs.

Predominant glucosinolates. The percentage differences of individual GSs indicate the type and quantity of predominant GSs within species and even within cultivars (Table 3). For example, gluconapin and gluconasturtiin were the predominant GSs in both cultivars of turnip, whereas glucobrassicin and neoglucobrassicin were the predominant GSs in Chinese cabbage 'Huangyacai' and 'Zaoshuwuhao', respectively. The major GSs in choysum 'Sijiu' were identified as sinigrin, gluconapin, glucobrassicanapin, and progoitrin, with the latter three generally predominating. In contrast, choysum 'Youqing' only contained two predominant GSs (gluconapin and glucobrassicanapin). From the results of Lewis and Fenwick (1988), glucobrassicanapin was predominant in Chinese cabbage and a mixture of gluconapin and glucoalyssin (not separated) were predominant GSs. He et al. (2002) also found that the predominant GSs were gluconapin and progoitrin in choysum and gluconapin and neoglucobrassicin in pakchoi. Hill et al. (1987) reported that the predominant GS was 3-butenyl-GS (gluconapin) in pakchoi and 3-indolylmethyl-GS (glucobrassicin) in Chinese cabbage and both 3-butenyl-GS and 3-indolylmethyl-GS in turnip. Except the variation in types of predominant GSs, there were differences in quantity of the same predominant GSs. For example, the relative content of predominant GS of gluconapin was $69 \%$ in purple cai-tai 'Wushihong', $40 \%$ to $53 \%$ in turnip, $31 \%$ to $39 \%$ in pakchoi, and $23 \%$ to $29 \%$ in choysum.

It was reported that there was great variation in GSs within different plant species (Mithen et al., 2000). Kang et al. (2006) reported that the genotypic effects described most of the phenotypic variation of GSs in Chinese cabbage than the environmental effects. In addition to genetic and environment factors, variation in GS types and concentrations among plant organs was reported in some Brassica vegetables (Bradshaw et al., 1983; Clossais-Besnard and Larher, 1991; Sang et al., 1984). The total amount of GSs was significantly less in the foliage than in the roots of turnip (Hill et al., 1987). The total content of GSs was highest in inflorescences followed by stems and leaves in choysum (He et al., 2000). In this study, three corresponding types of tissue (root, bolting stem, and leaves) were analyzed. The total GSs in roots of turnip are nearly two to three times larger than those in bolting stems and inflorescences of purple cai-tai and choysum and four to six times larger than those in leaves of chinese cabbage and pakchoi. Bolting stems and inflorescences of purple cai-tai and choysum mainly contained aliphatic GSs, in which relative content was $80 \%$ to $90 \%$, whereas the aromatic GSs were found in trace amounts only. In turnip roots, although the greatest GS group was aliphatic GSs $(60 \%$ relative to total GSs), there were also considerable aromatic GSs in which the relative content reached $28 \%$ to $35 \%$. The relative content of three principal GS groups was relatively equal in leaves of Chinese cabbage and pakchoi: $15 \%$ to $65 \%$ for the aliphatic group, $25 \%$ to $75 \%$ for the indolic group, and $8 \%$ to $15 \%$ for the aromatic group. Some research reports that differences in GS levels among plant organs were related to the ability of synthesis and storage of each organ (ClossaisBesnard and Larher, 1991; Rosa et al., 1996). 


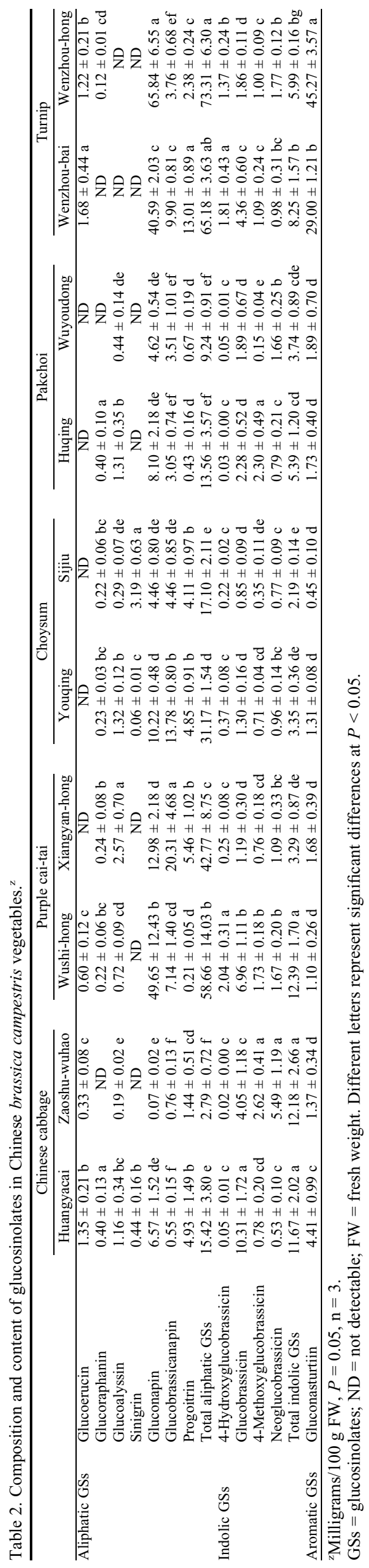

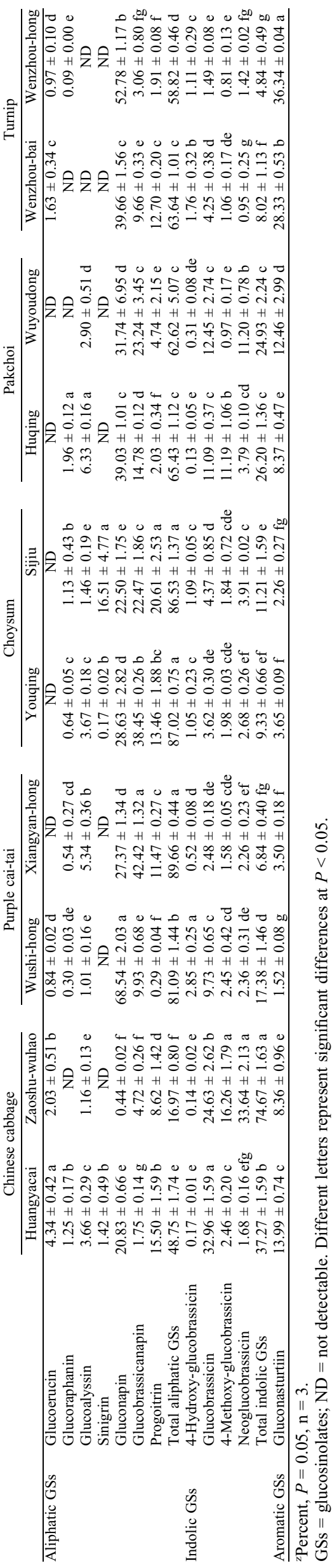

According to our data, roots of turnip had a stronger ability for synthesis and storage of GSs than stems and inflorescences of purple cai-tai and choysum; leaves of Chinese cabbage and pakchoi, especially on aromatic GSs; and bolting stems and inflorescences of purple cai-tai and choysum had preference on synthesis and storage of aliphatic GSs.

Detailed analysis from five Chinese Brassica campestris vegetables has shown considerable variation in total GS levels and in the content and relative content of individual GSs among species and among varieties within a species. The wide ranges of variation will offer important information for conventional breeding or genetic engineering with enhanced health benefits. The breakdown products from glucoraphanin, sinigrin, indole glucosinolates, and gluconasturtiin had been proven with potential beneficial effects on human health (Mithen et al., 2000). Considering these beneficial effects, Chinese cabbage, which contains high relative content of indolic GSs, and turnip, which contains high relative content of gluconasturtiin, are of high dietary value in all of these five Brassica campestris vegetables.

\section{Literature Cited}

Bradshaw, J.E., R.K. Heaney, G.R. Fenwick, and I.H. McNaughton. 1983. The glucosinolate content of the leaf and stem of fodder kale (Brassica oleracea L.), rape (Brassica napus L.) and radicole (Raphanobrassica). J. Sci. Food Agr. 34:571-575.

Carlson, D.J., M.E. Daxenbichler, C.H. VanEtten, W.F. Kwolek, and P.H. Williams. 1987. Glucosinolates in crucifer vegetables: Broccoli, brussels sprouts, cauliflower, collards, kale, mustard greens, and kohlrabi. J. Amer. Soc. Hort. Sci. 112:173-178.

Clossais-Besnard, N. and F. Larher. 1991. Physiological role of glucosinolates in Brassica napus. Concentration and distribution pattern of glucosinolates among plant organs during a complete life cycle. J. Sci. Food Agr. 56:25-38.

European Community. 1990. Determination of the oilseed glucosinolate content by HPLC. Off. J. Eur. Communities. L170: 03.07.27-34.

He, H., H. Chen, and W.H. Schnitzler. 2002 Glucosinolate composition and contents in Brassica vegetables. Sci. Agr. Sin. 35:192-197.

He, H., G. Fingerling, and W.H. Schnitzler. 2000 Glucosinolate contents and patterns in different organs of Chinese cabbages, Chinese kale (Brassica alboglabra Bailey) and choy sum (Brassica campestris L. ssp chinensis var. utilis Tsen et Lee). J. Appl. Bot.-Angew. Bot. 74:21-25.

Hill, C.B., P.H. Williams, D.G. Carlson, and H.L. Tookey. 1987. Variation in glucosinolates in Oriental Brassica vegetables. J. Amer. Soc. Hort. Sci. 112:309-313.

Kang, J.Y., K.E. Ibrahim, J.A. Juvik, D.H. Kim, and W.J. Kang. 2006. Genetic and environmental variation of glucosinolate content in Chinese cabbage. HortScience. 41:1382-1385.

Krumbein, A., I. Schonhof, and M. Schreiner. 2005. Composition and contents of phytochemicals (glucosinolates, carotenoids and chlorophylls) and ascorbic acid in selected Brassica species (B. juncea, B. rapa subsp. nipposinica var. chinoleifera, B. rapa subsp. Chinensis and B. rapa subsp. rapa). J. App. Bot. Food Qual. 79:168-174. 
Lewis, J. and G.R. Fenwick. 1988. Glucosinolate content of Brassica vegetables: Chinese cabbage pe-tsai (Brassica pekinensis) and pak-choi (Brassica chinensis). J. Sci. Food Agr. 45:379-386.

Mithen, R.F., M. Dekker, R. Verkerk, S. Rabot, and I.T. Johnson. 2000. Review: The nutritional significance, biosynthesis and bioavailability of glucosinolates in human foods. J. Sci. Food Agr. 80:967-984.
Mullin, W. and M. Sahasrabudhe. 1977. Glucosinolates content of cruciferous vegetable crops. Can. J. Plant Sci. 57:1227-1232.

Rangkadilok, N., M.E. Nicolas, R.N. Bennett, R.R. Premier, D.R. Eagling, and P.W.J. Taylor. 2002. Developmental changes of sinigrin and glucoraphanin in three Brassica species (Brassica nigra, Brassica juncea and Brassica oleracea var. italica). Sci. Hort. 96:11-26.
Rosa, E., R.K. Heaney, C.A.M. Portas, and G.R. Fenwick. 1996. Changes in glucosinolate concentrations in Brassica crops (B. oleracea and $B$. napus) throughout growing seasons. J. Sci. Food Agr. 71:237-244.

Sang, J., I. Minchinton, P. Johnstone, and R. Truscott. 1984. Glucosinolate profiles in the seed, root and leaf tissue of cabbage, mustard, rapeseed, radish and swede. Can. J. Plant Sci. 64:77-93. 